\title{
Evaluación de la coincidencia de los descriptores de las revistas españolas de PubMed
}

An assessment of descriptor coincidence in the Spanish journals of Pub-Med

\author{
Miguel Ángel Pellés García (1, 2), José Antonio Salvador Oliván (2)
}

(1) Biblioteca de Aragón, Zaragoza (España) (2) Departamento de Ciencias de la Documentación e Historia de la Ciencia. Universidad de Zaragoza. C/ Pedro Cerbuna, 12, 50009 Zaragoza (España); mapelles@unizar.es, jaso@unizar.es

\begin{abstract}
Resumen
Estudio que analiza la coincidencia en la indización que se da en revistas españolas en PubMed entre autores, indizadores profesionales y MeSH on Demand. El objetivo es determinar si la herramienta de indización automática es eficaz para la recomendación de términos de indización. Se extrajo una muestra representativa de 501 artículos sobre la población de revistas españolas ingresadas en PubMed en 2015 y se compararon artículo por artículo los descriptores asignados en cada caso por cada uno de los sujetos del estudio. Los resultados obtenidos demuestran que la coincidencia entre indizadores profesionales y $\mathrm{MeSH}$ on Demand es más alta que la obtenida por los autores. Así pues, se recomienda a las revistas científicas que animen a los autores a utilizar la herramienta de indización automática para la asignación de palabras clave con objeto de facilitar el acceso a la información.

Palabras clave: Indización. Grado de coincidencia en la indización. Indización automática. Métodos de indización. Medical Subject Headings (MeSH). MeSH on Demand. España.
\end{abstract}

\section{Introducción}

Actualmente tanto los productores de información científica, cómo las bases de datos más importantes de las distintas disciplinas destinan importantes recursos económicos y humanos a las tareas de indización de los documentos que componen sus colecciones. En esta línea y a modo de ejemplo, MEDLINE a comienzos de los 90 ya destinaba más de dos millones de dólares a estos menesteres (Hersh, Haynes y McKibbon, 1992). Una alternativa en la reducción de esos elevados costes de la indización humana viene de la mano de la indización automática. Esta variante al sistema de indización tradicional o humana viene siendo utilizada desde la década de los 60 por estos grandes productores de información científica (Gil Leiva y Rodríguez Muñoz, 1996).

Aunque no sin una cierta oposición al uso de la máquina para la realización de este tipo de tareas que desde tiempo remoto venían siendo desarrolladas por los profesionales de la información, se puede decir que hace ya tiempo, gracias al trabajo

\begin{abstract}
The study aims to compare the indexing consistency between authors, professional catalogers and $\mathrm{MeSH}$ on Demand in the Pubmed database. The objective is to identify if the automatic indexing tool is effective in recommending indexing terms. A representative sample of 501 papers published in Spanish journals in 2015 was extracted, and the three set of assigned descriptors were compared for each individual paper. The major findings of the study indicate that the consistency value between professional indexers and $\mathrm{MeSH}$ on $\mathrm{De}$ mand is higher than the value obtained by the authors. As a final conclusion, there is a need for scientific journals to recommend authors to use the automatic indexing tool when assigning keywords to their papers in order to facilitate access to information.
\end{abstract}

Keywords: Indexing. Degree of coincidence in indexing. Automatic indexing. Indexing methods. MeSH terms. MeSH on Demand. Spain.

de grupos multidisciplinares que tratan con las técnicas de procesamiento del lenguaje natural (PLN), se están desarrollando herramientas automáticas cada vez más complejas y precisas que siven para descargar de trabajo a estas secciones "humanas" de indización, o, al menos facilitar el trabajo tanto de los autores de textos científicos, como de los indizadores profesionales. En este sentido, en la US National Library of Medicine (NLM) se dan estos dos parámetros para desarrollar las tareas de indización de documentos: de un lado cuenta con una importante sección humana destinada estas tareas de indización de documentos; y de otro, dispone de una herramienta de indización automática que permite procesar para el indexado, los textos de los artículos biomédicos que ingresan en Medline, ofreciendo a autores o profesionales de PubMed, recomendaciones de términos para la indización de dichos textos.

A esta herramienta se le conoce como MeSH on Demand (Cho, 2014) y está accesible al público desde 2014. Su programa de procesamiento de textos se denomina Medical Text Indexer (MTI) 
(Aronson, 2004), está operativo desde 2002 y su uso se hace más intensivo en tareas de indización a partir de 2011. Dicha herramienta tiene su antecedente en el Proyecto Saphire (Hersh y Greenes, 1990), proyecto experimental para la indización automática y la recuperación de la información en Medline que se puso en funcionamiento en la década de los 90.

Son varios los estudios realizados sobre la coincidencia en la indización en la asignación de palabras clave, materias o descriptores a un documento (Abad García, Abad Pérez y Aleixandre Benavent, 1998; Moreno Fernández, Izquierdo Alonso y Vázquez Montávez, 2006; Evedove Tratarotti, Dal'Evedore y Lopes Fujita, 2017), pudiendo ser ésta realizada en periodos distintos o entre indizadores profesionales (Gil Leiva, 2002). La primera de ellas es denominada intraindización, y la segunda interindización; ambas se inscriben en la investigación de las diferencias individuales en la recuperación de la información (Saracevic, 1987) o, en otras palabras y fundamentalmente en el caso de la interindización, es una medida de la similitud de las reacciones de seres humanos diferentes cuando procesan la misma información (Zunde y Dexter, 1966).

Por tanto, este trabajo, aunque en cierta forma sigue la línea iniciada por Marcetich y Schuyler (1981), no pretende realizar un análisis de la coincidencia de la indización en sentido estricto. La finalidad del presente estudio es determinar el grado de coincidencia de los descriptores que asignan sujetos radicalmente diferentes (médicos, indizadores profesionales y una herramienta de indización automática); utilizando para ello herramientas de indización también diferentes; e incluso tomándose como base para la indización elementos distintos, ya que autor e indizadores de PubMed utilizan el texto completo; y para $\mathrm{MeSH}$ on Demand se ha utilizado el resumen elaborado por los propios autores.

Con este estudio se tratará de comprobar si la mencionada herramienta de indización automática permite, por un lado aconsejar de forma precisa a los autores de los trabajos médicos a la hora de elegir las palabras clave que acompañarán a sus artículos, y, por otro lado, ofrecer una lista adecuada de términos de cada artículo a los indizadores profesionales con el fin de poder ahorrar tiempo, y recursos a la NLM en estas tareas relativas a la indización.

\section{Método}

La población está constituida por los artículos publicados en revistas españolas indizados en Medline/PubMed en el año 2015. La estrategia de búsqueda utilizada para poder seleccionar posteriormente la muestra ha sido:

Spain [PL] AND 2015 [DP] AND 2015/01: 2015/12/31 [EDAD] AND medline [sb].

Los resultados obtenidos se filtraron por tipo de artículo, seleccionando de entre todos los disponibles los tres siguientes: Journal Article, Review y Systematic Reviews. El objetivo de este proceso de filtrado es dejar exclusivamente aquellos documentos que presentan una mayor posibilidad de inclusión de los términos de indización por parte de los profesionales de PubMed.

El resultado final de la búsqueda con su correspondiente filtrado fue de un total de 3009 artículos. Debido al número elevado de registros recuperados, se exportaron a 12 ficheros (uno por mes) en Refworks con el formato completo: título, fuente, autores, resumen y términos MeSH. Posteriormente, se exportaron a un único fichero Excel y de allí al programa estadístico SPSS, v. 20 para seleccionar la muestra. Se calculó el tamaño de la muestra que para un nivel de confianza del $95 \%$, un error de estimación del $4 \%$ y un valor desconocido de la $p$ y $q$, resultó en un total de 501 items. Los ítems se seleccionaron con el procedimiento de muestra aleatoria de casos del programa SPSS.

Las variables estudiadas, además de los datos obtenidos de los registros de Medline/PubMed fueron las siguientes: número de descriptores $\mathrm{MeSH}$; número de descriptores asignados por los autores; número de descriptores que proporciona $\mathrm{MeSH}$ on Demand; coincidencia entre los términos MeSH y los propuestos por los autores; coincidencia entre los términos MeSH y los asignados por MeSH on Demand; $y$ coincidencia entre los términos propuestos por los autores y los asignados por MeSH on Demand.

El trabajo de campo consistente en comparar los términos de indización asignados por autores de los artículos, indizadores profesionales de PubMed y la herramienta de indización automática $\mathrm{MeSH}$ on Demand se llevó a cabo según la metodología que se describe a continuación.

En primer lugar, se buscó el documento en PubMed a través del código PMID que tiene cada uno de los artículos ingresados en esta base de datos. Con el documento en pantalla se obtuvieron los siguientes datos: a) los términos MeSH asignados por los indizadores profesionales; b) el resumen del artículo incluido en la base de datos; y c) las palabras clave del artículo incluido en la base de datos. Se copió el resumen del documento, se pegó en la herramienta de indización automática MeSH on Demand y se procesó con objeto de obtener los descriptores generados a 
partir del proceso de indización automática. Finalmente, una vez obtenidos todos los parámetros se efectuó el análisis comparativo de los mismos teniendo en cuenta las siguientes circunstancias "especiales" y la solución determinada en cada caso.

El autor principal comprobó si los descriptores coincidían entre los distintos procedimientos de indización, y descartó los registros problemáticos.

En primer lugar, no en todos los ítems de la muestra se puede llevar a cabo el contraste entre descriptores. El motivo es que, o bien faltan las palabras clave en el original, o bien falta el resumen; e incluso ambos en alguno de los ítems. El total de ítems incompletos en alguno de los citados elementos es de 89 sobre un total de 501 registros, lo que hace que casi un $17,8 \%$ de la muestra quede fuera del estudio.

Por otra parte, hay autores que usan descriptores que coinciden en su contenido, pero los equivocan en su forma, afectándolos en aspectos como el orden de los términos y sus correspondientes inversiones cuando se trata de descriptores compuestos, las palabras incluidas en el descriptor, etc. También hay problemas en el número gramatical de los descriptores; por ejemplo, el autor emplea un singular cuando el descriptor va en plural o a la inversa. En estos casos, se decidió no considerarlos como coincidentes.

Además, hay que mencionar también algunos cambios producidos en la nueva versión (julio de 2017) de MeSH on Demand (MeSH on Demand Update, 2017) que afectan a las pautas en el trabajo de campo. En primer lugar, la nueva versión no informa del número de caracteres del texto ingresado, aspecto que se estaba recogiendo hasta el momento en el estudio con objeto de comprobar si el tamaño del texto ingresado es directamente proporcional al número de términos extraídos. El problema se solventó pasando el texto por un contador de caracteres y palabras gratuito y así se recupera dicho dato - concretamente http://www.contadordecaracteres.com/- . En segundo lugar, se ha variado el orden el que presentan los descriptores obtenidos. En la versión anterior se presentaban en orden alfabético y ahora en función de la relevancia del término. Esto afecta a la rapidez de contraste entre los descriptores que dan los indizadores de PubMed (expuestos en orden alfabético) y los que ofrece la herramienta. Finalmente, otro cambio relevante afecta a los descriptores más "básicos" destinados a delimitar lo que se puede denominar como "objeto de estudio", que en general son "menos importantes", como ocurre con los identificadores del tipo de grupo al que va dirigido el estudio Humans, Animals, Male, Female, etc. son ahora calificados como Additional Terms. Como hasta ahora no se hacía esta distinción se decide computarlos como si estuvieran integrados dentro de la sección de términos MeSH. Tanto en lo que atañe en el cómputo global de términos que ofrece dicha herramienta, como a la hora de contrastar la información dada con los términos $\mathrm{MeSH}$ que dan los indizadores profesionales.

En cuanto al proceso y análisis de los datos, el análisis estadístico se realizó con el programa SPSS. Se obtuvo la estadística descriptiva de cada variable y se analizó la correlación mediante el coeficiente de Pearson. Para las comparaciones se utilizó el análisis de la varianza para medidas repetidas.

\section{Resultados}

El número total de artículos estudiados ha sido de 501 distribuidos en 54 revistas. Las revistas con mayor número de artículos en la muestra seleccionada para el trabajo son: Nutricion hospitalaria con un total de 94 artículos analizados, Cirugia y cirujanos con un total de 24 artículos analizados, Revista de neurología con un total de 23 artículos analizados y Histology and histopathology con un total de 21 artículos analizados. Estas cuatro revistas comprenden el $32.3 \%$ de los artículos estudiados, frente al $67.7 \%$ restante distribuido entre las 50 revistas restantes.

\subsection{Número de descriptores}

El número de términos MeSH por artículo asignados por los profesionales que indizan artículos en PubMed y los asignados por la herramienta $\mathrm{MeSH}$ on Demand, es muy similar, obteniendo en ambos casos una media de 12,6 descriptores. Sin embargo, los autores de los artículos incluyen una media de 4,5 descriptores, un dato muy inferior.

\begin{tabular}{lrrr}
\hline & Media & Moda & Rango \\
\hline Profesionales & 12,6 & 12 & $2-33$ \\
\hline MeSH on Demand & 12,6 & 12 & $3-38$ \\
\hline Autores & 4,5 & 4 & $1-13$ \\
\hline
\end{tabular}

Tabla I. Estadística descriptiva de descriptores

La moda obtenida también es coincidente en el trabajo realizado por los indizadores profesionales y por la herramienta de indización automática, frente a los autores de los textos. En este sentido el número de descriptores asignados a cada uno de los artículos por cada uno de los tres elementos estudiados, muestra que las cifras más repetidas de términos incluidos en un artículo son 12 
términos para indizadores profesionales y $\mathrm{MeSH}$ on Demand y 4 términos para los autores.

En cuanto al número máximo y mínimo de descriptores asignados a cada artículo por parte de las diferentes variables estudiadas decir que el rango entre indizadores profesionales y herramienta es prácticamente coincidente, se está en ambos casos ante una horquilla muy amplia y similar, mientras que dicho rango es mucho menor cuando se trata de los autores.

En la Tabla II se presentan las diferencias entre los tres elementos estudiados. El número de descriptores utilizado por los profesionales es prácticamente el mismo que ofrece la herramienta $\mathrm{MeSH}$ on Demand; sin embargo, sí hay diferencias significativas cuando se comparan con el número de palabras clave empleadas por los autores, con un promedio de 8 descriptores más.

\begin{tabular}{lccc}
\hline & $\begin{array}{c}\text { Dif. } \\
\text { medias }\end{array}$ & $\begin{array}{c}\text { IC 95\% } \\
\text { diferencia }\end{array}$ & $p^{a}$ \\
\hline $\begin{array}{l}\text { Profesionales - } \\
\text { MeSH on Demand }\end{array}$ & $-0,29$ & $-0,77-0,71$ & 1,00 \\
\hline $\begin{array}{l}\text { Profesionales - } \\
\text { Autores }\end{array}$ & 8,05 & $7,44-8,66$ & $<0,001$ \\
\hline $\begin{array}{l}\text { MeSH on Demand } \\
- \text { Autores }\end{array}$ & 8,08 & $7,51-8,66$ & $<0,001$ \\
\hline
\end{tabular}

Tabla II. Comparación de descriptores en los tres casos

(Dif. Medias: Diferencia de medias. IC: Intervalos de confianza. ${ }^{a}$ Valor de la $p$ para el test de MANOVA para medidas repetidas)

Hay evidencia también de una relación lineal positiva moderada entre el número de descriptores asignados por los profesionales y el de $\mathrm{MeSH}$ on Demand ( $r=0,161, p=0,001)$, asociándose en ambos casos un mayor número de términos con una mayor longitud del resumen, aunque la intensidad de esta relación es mayor con $\mathrm{MeSH}$ on $\mathrm{De}$ mand $(r=0,474, p=0,001$ vs $r=0,216, p=0,001)$.

\subsection{Análisis de coincidencia de descriptores}

En este apartado se analiza el grado coincidencia entre los descriptores asignados a los artículos por los profesionales de la indización, autores y la herramienta de indización automática.

El grado de coincidencia entre los términos dados por los autores de los artículos y los términos dados por los indizadores profesionales de PubMed es de $21,1 \%$. Solo hay 8 artículos que presentan una coincidencia del $100 \%$, lo que supone el $1,9 \%$ del total, mientras que la coincidencia es nula (0\%) en 162 artículos (39,3\% de la muestra).
El grado de coincidencia entre los términos dados por los autores de los artículos y la herramienta de indización automática es muy bajo, del $8,1 \%$, siendo nula en 166 artículos y no hay ningún artículo donde sea total.

\begin{tabular}{lrr}
\hline & \multicolumn{1}{c}{ Media } & \multicolumn{1}{c}{$\begin{array}{c}\text { IC 95\% } \\
\text { diferencia }\end{array}$} \\
\hline Autores - Profesionales & $21,1 \%$ & $18,9 \%-23,2 \%$ \\
\hline Autores - MeSH on Demand & $8,1 \%$ & $7,2 \%-9,0 \%$ \\
\hline $\begin{array}{l}\text { MeSH on Demand - } \\
\text { Profesionales }\end{array}$ & $45,9 \%$ & $44,0 \%-47,7 \%$ \\
\hline
\end{tabular}

Tabla III. Comparación de descriptores entre los tres elementos

El grado de coincidencia entre los términos dados por los indizadores profesionales de PubMed y los términos dados por $\mathrm{MeSH}$ on Demand es del $45,9 \%$. Solo hay 2 artículos en los que la coincidencia es completa, y otros 2 en los que no coindice ningún término.

Cabe destacar que el grado de coincidencia en los términos asignados para cada artículo entre profesionales de la indización y la herramienta de indización automática es muy superior a cualquiers de las otras dos situaciones, quedando como menos coincidentes los términos asignados entre los autores de los artículos y la herramienta de indización automática. Hay que resaltar que esta alta tasa de coincidencia entre los indizadores profesionales y la herramienta de indización automática se produce en términos de indización básicos, es decir, aquellos que ayudan a delimitar el tema objeto de estudio del artículo.

Algunas cuestiones significativas que afectan a la asignación de encabezamientos básicos son los siguientes: a) la identificación del fin o el objeto del estudio: si está dirigido a humanos o animales, o por diferencias de género entre hombres y mujeres, etc.; y b) aludiendo a estudios concretos vinculados a una forma específica o a un tipo de estudio específico, como por ejemplo si son encuestas o cuestionarios, estudios prospectivos o estudios retrospectivos, etc.

Sin embargo y en la línea propuesta en el punto anterior relativa a la coincidencia de términos básicos, hay que decir que también se observan diferencias en la asignación de este tipo de descriptores entre los indizadores expertos de PubMed y la herramienta de indización automática. Esta circunstancia se ve por ejemplo en el caso de la asignación de descriptores referidos al grupo de edad al que afecta el estudio realizado en el artículo, es decir, si se trata de un estudio 
cuyo sujeto son niños, jóvenes, adultos o personas mayores. Mientras que los indizadores profesionales sí que proporcionan esta información detallando ese grupo de edad sobre el que se efectúa el estudio, de forma general se puede decir que MeSH on Demand obvia este tipo de información y no asigna este tipo de descriptores o lo hace en muy pocas ocasiones.

Otro aspecto a destacar es que hay gran coincidencia entre los indizadores profesionales y la herramienta automática cuando en el artículo se trata una enfermedad específica y concreta, como un síndrome con un nombre concreto (por ej. Metabolic Syndrome $X$ ) o similares. En general $\mathrm{MeSH}$ on Demand identifica bien este tipo de situaciones y tiene una tasa de coincidencia elevada con los indizadores profesionales.

En cuanto a los aspectos negativos de la herramienta de indización automática, se observa una circunstancia que tiene que ver con la incoherencia en la selección de términos por parte de $\mathrm{MeSH}$ on Demand. Esta incoherencia se ve, por ejemplo, en artículos vinculados al tipo de sujeto sobre el que versa el estudio. Por ejemplo, a un estudio hecho sobre ratas (es decir, dentro de la categoría de 'Animales'), MeSH on Demand le asigna también el descriptor 'Humanos', es decir, en un estudio adscrito a la categoría de animales, la herramienta asigna los dos descriptores, antagónicos entre sí. Esto no ocurre prácticamente nunca en los descriptores asignados por los profesionales, en los que se mantiene la coherencia en todos los casos. Un ejemplo de esta circunstancia se ve en los artículos con PMID 26274892 o y 26238592.

A continuación, se presentan las revistas españolas en las que existe mayor y menor coincidencia en la asignación de descriptores:

- Coincidencia mayor del 90\% entre autores e indizadores profesionales de PubMed. Tras el estudio se encuentran un total de 8 artículos distribuidos en 6 títulos de revista en los que la coincidencia entre los términos dados por los autores de los artículos y los términos dados por los indizadores profesionales es mayor del $90 \%$. Siendo la revista Nutrición hospitalaria con 3 artículos la que mayor concordancia ha alcanzado en esta comparativa.

- Coincidencia mayor del 90\% entre autores y $\mathrm{MeSH}$ on Demand. No se obtienen resultados en esta faceta, es decir, en ningún artículo se alcanza una tasa de coincidencia superior al $90 \%$ entre los términos asignados por los autores de los artículos y la herramienta de indización automática.
- Coincidencia mayor del $90 \%$ entre indizadores profesionales de PubMed y $\mathrm{MeSH}$ on Demand. Tras el estudio, se encuentran un total de 3 artículos distribuidos en 3 títulos de revista, en los que la coincidencia entre los términos dados por los indizadores profesionales y los términos dados por la herramienta de indización automática es mayor del $90 \%$. Las revistas que mayor concordancia ha alcanzado en esta comparativa son Psicothema, Reumatología clínica y la Revista Española de Sanidad Penitenciaria.

- Coincidencia menor del $10 \%$ entre autores e indizadores profesionales de PubMed. El estudio detalla que hay un total de 164 artículos distribuidos en 42 títulos de revista en los que la coincidencia entre los términos dados por los autores de los artículos y los términos dados por los indizadores profesionales es menor del $10 \%$. Los títulos de revista con mayor número de artículos con esta escasa coincidencia entre las palabras clave asignadas por los dos entes objeto de estudio para este caso son Nutrición hospitalaria (con 23 artículos); Revista de neurología (con 12 artículos) y; Cirugía y cirujanos (con 11 artículos).

- Coincidencia menor del $10 \%$ entre autores y MeSH on Demand. Hay un total de 265 artículos distribuidos en 49 títulos de revista en los que la coincidencia entre los términos dados por los autores de los artículos y los términos asignados por la herramienta de indización automática es menor del $10 \%$. Los títulos de revista con mayor número de artículos con esta escasa coincidencia entre las palabras clave asignadas por los dos entes objeto de estudio para este caso son Nutrición hospitalaria (con 56 artículos); Cirugía y cirujanos (con 17 artículos); Revista de neurología (con 15 artículos); Archivos Españoles de Urología (con 12 artículos) y; Journal of Physiology and Biochemistri (con 10 artículos).

- Coincidencia menor del $10 \%$ entre indizadores profesionales de PubMed y $\mathrm{MeSH}$ on Demand. El estudio revela que hay un total de 5 artículos distribuidos en 5 títulos de revista en los que la coincidencia entre los términos dados por los indizadores profesionales de PubMed y los términos asignados por la herramienta de indización automática es menor del $10 \%$.

\section{Conclusiones}

Tras el trabajo de campo y el análisis de los resultados obtenidos en él se extraen las siguientes conclusiones: 
1. El número medio de descriptores asignados por artículo es muy bajo cuando se trata de las palabras clave que dan los autores para su artículo. Con una media de 4 descriptores por artículo, frente a la media de 12 que dan tanto indizadores profesionales como $\mathrm{MeSH}$ on Demand, se determina como una seria traba de cara a la recuperación de la información, pues ese menor número de descriptores asignados por autores a los artículos reduce las posibilidades a la hora de recuperar los artículos a través de su contenido. A tenor de los resultados, si los autores emplearan la herramienta de indización, ésta les recomendaría un mayor número de descriptores y por tanto se incrementarían las posibilidades de recuperar la información.

2. El grado medio de coincidencia alcanzada entre indizadores profesionales y $\mathrm{MeSH}$ on $\mathrm{De}$ mand es más del doble que la media de cualquiera de las combinaciones en las que participan los autores como sujeto de comparación en dicha coincidencia en la indización. Si se da como premisa que la indización óptima es la efectuada por los indizadores profesionales de PubMed, es decir, se considera que los profesionales asignan los términos con mayor precisión en relación con el contenido del documento, es altamente recomendable que los autores utilicen la herramienta de indización automática, con lo que mejoraría la calidad de la indización y redundaría en una más eficaz recuperación de la información (Gil Leiva, 2008).

3. La herramienta de indización automática evita en la mayoría de las ocasiones los errores de número o forma en los descriptores elegidos por parte de los autores. Errores que tienen que ver con la asignación de palabras clave en las que equivocan el número, dando un plural en vez de un singular o a la inversa; y errores de forma que tienen que ver con la inversión de términos, la elección de una sigla en vez de la forma desarrollada o la inversa, etc. La herramienta de indización automática no falla nunca en este tipo de cuestiones al recomendar los términos de indización. El acertar en estos términos, en ocasiones muy relevantes por ser la materia principal sobre la que versa el artículo, elevaría el grado de coincidencia en la indización favoreciendo de nuevo la recuperación de la información.

A tenor de lo expuesto, decir que $\mathrm{MeSH}$ on $\mathrm{De}$ mand es una herramienta de indización automática que, a pesar de las constantes mejoras que ha recibido en los últimos años, sigue presentando problemas en las recomendaciones de descriptores $\mathrm{MeSH}$ que hace a sus usuarios.
No obstante, tasas como la más que aceptable coincidencia en la indización en la pareja conformada por indizadores profesionales y la propia herramienta, demuestran que es una herramienta bastante eficaz en la tarea de indización, que además de rapidez en este trabajo, mejora con mucho el trabajo realizado por parte de los autores en la asignación de las palabras clave que acompañan a sus artículos.

Las conclusiones a las que se llega tras este estudio confluyen en dos sugerencias básicas. La primera de ellas es que las editoriales o revistas científicas españolas responsables de los artículos albergados en PubMed recomienden a sus autores extraer las palabras clave del resumen que acompaña a su artículo, filtrando dicho artículo a través de la herramienta de indización automática MeSH on Demand.

El uso de $\mathrm{MeSH}$ on Demand en el proceso de asignación de palabras clave no solo facilita y mejora la calidad de los descriptores, sino que también asigna de forma muy sencilla información relevante sobre el propio artículo que los autores no suelen incluir a través de sus palabras clave: sobre el fin o el objeto de estudio, del tipo de estudio que es, a qué grupo de personas va dirigido, etc. La herramienta de indización automática sí que asigna términos en esta línea permitiendo a un usuario final, conocer el tipo de artículo ante el que se encuentra o cuestiones particulares sobre las que versa dicho artículo.

La segunda de las sugerencias va dirigida al equipo de indizadores profesionales de Medline/ Pubmed, invitándoles a depositar parte de su confianza en los términos MeSH ofrecidos de forma automática por MeSH on Demand, puesto que a partir del resumen del artículo y en función de su extensión y de la corrección del mismo, se permite ver de forma muy rápida cuál es el tema central sobre el que versa el documento y cuáles son algunos de los términos de delimitación más importantes del objeto de estudio. Como ya se ha dicho anteriormente, dichos términos son solo recomendaciones y quedarían a expensas de un posterior tratamiento humano con el fin de subsanar posibles errores de la herramienta de indización automática. No obstante como se puede ver tras los datos obtenidos en este estudio, el uso de MeSH on Demand permitiría al menos orientar a los profesionales de la indización de Medline/Pubmed en su trabajo diario.

\section{Referencias}

Abad García, M. F.; Abad Pérez, I.; Aleixandre Benavent, R. (1998). Evaluación de la consistencia en la indización del reper-torio Documentación Médica Española. // Revista Española de Documentación Científica. 21:4, 389-401. 
Aronson, A. R.; y otros (2004). The NLM Indexing Initiative's Medical Text Indexer // Medinfo (sept.). 268-272.

Cho, D. (2014). MeSH on Demand Tool: An Easy Way to Identi-fy Relevant MeSH Terms. // NLM Technical Bulletin. 398. https://www.nlm.nih.gov/pubs/techbull/mj14/mj 14_MeSH_on_demand.html (may-june) (2018-01-12).

Dal' Evedove Tartarotti, R. C.; Dal' Evedore, P.R.; Spotti Lopes, M. (2017). Avaliação Da Consistência Da Indexação Em Biblio-tecas Universitárias Federais Da Região Nordeste do Brasil. // Anales De Documentación. 20:1, 1-19. https:// doi.org/10.6018/analesdoc.20.1.253201 (2018-01-12).

Hersh, W. R.; Greenes, R. A. (1990). Saphire: An information retrieval system featuring concept matching, automatic indexing, probabilistic retrieval, and hierarchical relationships. // Computers and Biomedical Research. 23:5, 410-425.

Hersh, W.R.; Haynes, R. B.; McKibbon, K. A. (1992). Evaluation of SAPHIRE: an automated approach to indexing and retrieving medical literature. // Proceedings of the Fifteenth Annual Symposium on Computer Applications in Medical Care: A Conference of the American Medical Informatics Association. 808-12.

Gil Leiva, I. (2002). Consistencia en la indización de documen-tos entre indizadores noveles. // Anales de documentación. 5, 99-111.

Gil Leiva, I. (2008). Manual de indización: Teoría y práctica. Gijón: Trea.

Gil Leiva, I.; Rodríguez Muñoz, J. V. (1996). Tendencias en los sistemas de indización automática: Estudio evolutivo. // Revista Española de Documentación Científica. 19:3, 273-291.
Marcetich, J.; Schuyler, P. (1981). The use of AID to promote indexing consistency at the National Library of Medicine. // Eight-first Annual Meeting of the Meddical Library Association. Montreal: Medical Library Association.

MeSH on Demand Update (2017). // NLM Technical Bulletin. 416 (may-june). https://www.nlm.nih.gov/pubs/techbull/mj 17/mj17_MeSH_on_demand_update.html (2018-01-12).

Moreno Fernández, L. M.; Izquierdo Alonso, M.; Vázquez Montávez, A. J. (2006). La consistencia como indicador de la fiabilidad (corrección) de la indización: los casos de LISA, PASCAL y VOCED. // Scire. 12:1 (en.-jun. 2006) 85-98.

Mork, J.G.; Jimeno Yepes, A.J..; Aronson, A.R. (2013). The NLM medical text indexer system for indexing biomedical literature. // BioASQ Workshop. Valencia. http://bioasq.org/sites/default/files/Mork.pdf (2018-01-12).

Saracevic, T. (1991). Individual differences in organizing, searching and retrieving information. // Proceedings of the 54TH Annual Meeting of the American Society of Information Science. 82-86.

Stevens, M.E. (1965). Automatic indexing: a state of the art report, Monograph 91. National Bureau of Standars, Washington.

Zunde, P.; Dexter, M. (1969). Indexing consistency and quality. // American Documentation. 20:3. 259-267.

Enviado: 2018-03-20. Segunda versión: 2019-05-02. Aceptado: 2019-05-02. 
